\title{
Rechtsprechung
}

\section{Eintragung des von George Grosz stammenden Werks „Brillantenschieber im Café Kaiserhof" in das Verzeichnis national wertvollen Kulturgutes}

\author{
OVG Berlin-Brandenburg, Beschluss vom 12. April 2019 - OVG 10 N 54.17
}

\section{Tenor}

Das Berufungszulassungsverfahren wird eingestellt, soweit der Kläger als Rechtsnachfolger seiner Mutter I... begehrt, das Urteil des Verwaltungsgerichts Berlin zu ändern und den Bescheid vom 22. Juni 2011 insoweit aufzuheben, als damit die Kunstwerke „Belebte Straßenszene" und "Schönheit, Dich will ich preisen“ von George Grosz, „Ertüchtigung" von Hannah Höch, „Zwischen Bäumen stehendes Mädchen“ von Otto Mueller sowie "Zwei nackte Tanzende“ und „Mädchen auf violettem Sessel“ von Ernst Ludwig Kirchner in das Verzeichnis national wertvollen Kulturgutes eingetragen werden.

Im Übrigen wird der Antrag des Klägers auf Zulassung der Berufung gegen das Urteil des Verwaltungsgerichts Berlin vom 22. Januar 2015 abgelehnt.

Die Kosten des Berufungszulassungsverfahrens trägt der Kläger.

Der Streitwert wird für die zweite Rechtsstufe auf 2.125.000,00 EUR festgesetzt, wobei auf den erledigten Teil des Berufungszulassungsverfahrens 1.821.000,00 EUR entfallen.

\section{Gründe}

I.

[1] Der Kläger ist Rechtsnachfolger seiner verstorbenen Eltern F... und I...leitete die Berliner Galerie N..., zu deren Betriebsvermögen u.a. die Kunstwerke „Belebte Straßenszene “, "Schönheit, Dich will ich preisen" und „Brillantenschieber im Café Kaiserhof" von George Grosz, „Ertüchtigung “ von Hannah Höch, "Zwischen Bäumen stehendes Mädchen“ von Otto Mueller sowie „Zwei nackte Tanzende" und "Mädchen auf violettem Sessel“ von Ernst Ludwig Kirchner gehören. Am 22. Juni 2011 erließ der Beklagte einen an die Eltern des Klägers adressierten Bescheid, mit dem die Eintragung u.a. der zuvorerwähnten Kunstwerke in das Verzeichnis national wertvollen Kulturgutes aufder Grundlage des $\S 1$ Abs. 1 S. 1 des Gesetzes zum Schutz deutschen Kulturgutes gegen Abwanderung vom 8. Juli 1999 (BGBI. I S. 1755; im Folgenden: KultgSchG) verfügt wurde. Hiergegen erhoben die Eltern des Klägers Klage. Nachdem die Mutter des Klägers in dem Klageverfahrenverstorben war, nahmen der Kläger und dessen Vater als Rechtsnachfolger der Mutter des Klägers das Verfahren auf.
[2] Das Verwaltungsgericht Berlin hat mit Urteil vom 22. Januar 2015 den Bescheid des Beklagten vom 22. Juni 2011 insoweit aufgehoben, als damit die Kunstwerke "Belebte Straßenszene" und "Schönheit, Dich will ich preisen“ von George Grosz, „Ertüchtigung" von Hannah Höch, "Zwischen Bäumenstehendes Mädchen" von Otto Mueller sowie "Zwei nackte Tanzende" und „Mädchen auf violettem Sessel“ von Ernst Ludwig Kirchner in das Verzeichnis national wertvollen Kulturgutes eingetragen werden. Im Übrigen hat es die Klage abgewiesen. Dabei hat es die Klage wegen fehlender Klagebefugnis bereits fürunzulässig erachtet, soweit der Kläger und dessen Vater ihre (jeweilige) Klageals Rechtsnachfolger der Mutter der Kläger erhoben haben. Gegen dieses Urteil richtet sich der Antrag des Klägers auf Zulassung der Berufung, der das Verfahren auch als Rechtsnachfolger seines mittlerweile verstorbenen Vatersaufgenommen hat.

[3] Der Kläger - seinerzeit noch als Kläger zu 2. - hat das Berufungszulassungsverfahren noch vor dem Tod seines Vaters für erledigterklärt, „soweit die Änderung des Urteils und Aufhebung des Bescheides des Beklagten vom 22. Juni 2011 hinsichtlich der dort genannten Werke -ausgenommen das Werk (von) George Grosz, "Brillantenschieber im CaféKaiserhof" - begehrt wird. Der Beklagte hat sich der Erledigungserklärung des Klägers angeschlossen.

\section{II.}

[4] 1. Das Berufungszulassungsverfahren war, soweit es von den Beteiligten in der Hauptsache für erledigt worden ist, in entsprechender Anwendung des $\S 92$ Abs. 3 in Verbindung mit $\S$ 125 Abs. 1 S. 1 VwGO einzustellen.

[5] 2. Im Übrigen hat der Antrag auf Zulassung der Berufung keinen Erfolg. Die

geltend gemachten Berufungszulassungsgründe der ernstlichen Zweifel ander Richtigkeit des Urteils (§ 124 Abs. 2 Nr. 1 VwGO), der grundsätzlichen Bedeutung der Rechtssache ( $\S$ 124 Abs. 2 Nr. 3 VwGO), der Divergenz (§ 124 Abs. 2 Nr. 4 VwGO sowie des Verfahrensmangels ( $\$ 124$ Abs. 2 Nr. 5 VwGO) liegen nicht vor. Maßgebend für die Prüfung des Oberverwaltungsgerichts sind allein die dargelegten Gründe (§ 124a Abs. 4 S. 4 VwGO). Diese vermögen die Zulassung der Berufung nicht zu rechtfertigen. 
[6] a) Ernstliche Zweifel an der Richtigkeit der angegriffenen Entscheidung im Sinne des § 124 Abs. 2 Nr. 1 VwGO zeigt der Kläger nicht auf. Derartige Zweifel sind nicht erst dann gegeben, wenn bei der im Zulassungsverfahrenallein möglichen summarischen Prüfung der Erfolg des Rechtsmittelswahrscheinlicher ist als der Misserfolg; denn das Zulassungsverfahren hatnicht die Aufgabe, das Berufungsverfahren vorwegzunehmen. Ernstliche Zweifel sind schon dann anzunehmen, wenn ein einzelner tragender Rechtssatz oder eine erhebliche Tatsachenfeststellung mit schlüssigen Argumenten in Frage gestellt wird (vgl. BVerfG, Beschluss vom 6. Juni 2018 -2 BvR 350/18 -, juris Rn. 16; ebenso Senatsbeschluss vom 29. Januar 2019-OVG 10 N 67.16 -, juris Rn. 8). Das ist hier nicht der Fall.

[7] aa) Die Kritik des Rechtsbehelfs gegen die Annahme des Verwaltungsgerichts, die Klage des Klägers (in seiner vormaligen prozessualen Rolle als Kläger zu 2.) sei bereits unzulässig, überzeugt nicht.

[8] (1) Das Verwaltungsgericht hat seine Ansicht wie folgt begründet: Es fehle an der gem. § 42 Abs. 2 VwGO erforderlichen Klagebefugnis, die bereits die Erblasserin (d.h. die Mutter des Klägers) nicht habe geltend machen können.Ihre Verletzung in eigenen Rechten erscheine nicht als möglich. Die belastende Wirkung der angefochtenen Eintragungsverfügung bestehe inerster Linie in dem Ausfuhrgenehmigungsvorbehalt gem. § 1 Abs. 4 S. 1 KultgSchG, der das durch Art. 14 Abs. 1 S. 1 GG gewährleistete Nutzungsrecht des Eigentümers oder eines mit aus dem Eigentumsrechtfließenden Verfügungsbefugnissen ausgestatteten Nichteigentümers beschränke. Dass die Erblasserin eine Rechtsposition innegehabt hätte, aus der sich eine derartige Belastung für sie hätte ergeben können, sei nicht dargetan. Vielmehr sei sie nach dem schriftsätzlichen Vorbringen des Klägers und seines Vaters weder Eigentümerin der streitgegenständlichen Kunstwerke noch sonst verfügungsberechtigt gewesen. Dafür spreche auch der Umstand,dass Partei des rechtskräftig abgeschlossenen Zivilrechtsstreits, der beim Kammergericht unter dem Aktenzeichen - 20 U 110/08 geführt worden sei, einzig der Vater des Klägers gewesen sei. Insoweit hätten durch den Erbfallauch keine Rechte auf den Kläger übergehen können. Soweit der Klägervertreter - entgegen dem ursprünglichen Vortrag - in der mündlichen Verhandlung ausgeführt habe, es sei zwar unwahrscheinlich, könne aber auch nicht ausgeschlossen werden, dass die Mutter des Klägers (Mit-)Eigentümerin gewesen sei, genüge dies nicht, um eine Klagebefugnis zu begründen. Denn der Kläger sei hinsichtlich der zivilrechtlichen Eigentumsverhältnisse darlegungs- und beweisbelastet. Auch die gegebenenfalls fehlerhafte Adressierung des angefochtenen Bescheides vom 22. Juni 2011 an I... und F... vermöge die Klagebefugnis des Klägers nicht zu begründen. Die aus Art. [2] Abs. 1 GG abgeleitete sog. Adressatentheorie begründe lediglich die Vermutung, dass eine Verletzung der allgemeinen Freiheitsgewährleistung nach Art. 2 Abs. 1 GG in Betracht komme. Diese Vermutung sei vorliegend jedoch entkräftet, da die Annahme einer belastenden Wirkung hinsichtlich der Mutter und des Klägers (als ihres Rechtsnach- folgers) ausscheide. Eine Klagebefugnis könne hier auch nicht deshalb angenommen werden, weil -angesichts der gegebenenfalls fehlerhaften Adressierung des Verwaltungsaktes - ein Bedürfnis zur Beseitigung eines etwaigen „Rechtsscheins“ zu Lasten der Erblasserin bestehe. Zum einen könnte sich insoweit allenfalls ein Anspruch auf Berichtigung des Verwaltungsaktes, nicht aber der vorliegend auch durch den Kläger (als Rechtsnachfolger seiner Mutter) geltend gemachte Aufhebungsanspruch ergeben. Zum anderen wirke ein etwaiger belastender Rechtsschein, der einzig die Erblasserin betroffen habe, gegenüber dem Kläger nicht mehr fort (vgl. S. 23 f. des Urteils; in juris abgedruckt unter Rn. 57 f.).

[9] (2) Der Kläger unterlässt es, sich mit diesen Erwägungen näher auseinanderzusetzen, weil er meint, das Verwaltungsgericht habe verkannt, dass der Beklagte in dem angefochtenen Bescheid wie auch in dem Verfahren davor und in der Zeit danach immer angenommen habe, die Mutter des Klägers sei (Mit-)Eigentümerin bzw. neben dem Vater des Klägers eine weitere dingliche Verfügungsberechtigte der bzw. über die von der Eintragungsverfügung betroffenen Kunstwerke - einschließlich des hier noch allein verfahrensgegenständlichen Bildes von George Grosz - gewesen. Diese Argumentation wie auch die für sie klägerseits angeführten Argumente verfangen nicht. Im Einzelnen:

[10] (a) Die Anfechtungsklage nach § 42 Abs. 2 VwGO ist nur zulässig, wenn der Kläger geltend macht, durch den angefochtenen Verwaltungsakt in seinen Rechten verletzt zu sein. Dafür genügt es, wenn die Möglichkeit der vom Kläger behaupteten Rechtsverletzung besteht; das ist nicht der Fall, wenn offensichtlich und eindeutig nach keiner Betrachtungsweise Rechte des Klägers verletzt sein können (stRspr., s. nur BVerwG, Urteil vom 24. Mai 2018 - BVerwG 3 C 18.16 -, juris Rn. 9). Ausgehend von dem bisherigen Vorbringen des Klägers und seiner Rechtsvorgängerin erscheint eine Verletzung seiner bzw. ihrer Rechte nicht möglich.

[11] (b) Der Hinweis des Klägers, dass der streitgegenständliche Bescheid an seine Mutter gerichtet sei, muss unergiebig bleiben.

[12] Dass die Rechtsvorgängerin des Klägers Adressatin des angefochtenen Bescheides ist, verhilft dem Rechtsbehelf nicht zum Erfolg. Nach der -klägerseits wohl ohnehin nicht als tragfähige Grundlage für die Annahme einer Klagebefugnis erachteten - Adressatentheorie ist zwar derjenige klagebefugt, an den sich die in dem Bescheid getroffene Regelung richtet (vgl. BVerwG, Beschluss vom 18. Juni 2014 - BVerwG 3 B 28.14 -, juris Rn. 11). Im Einzelfallkann der Adressat eines Verwaltungsaktes aus dieser Theorie allerdings dannkeine Klagebefugnis für sich herleiten, wenn er zwar Bekanntgabeadressat,nicht aber zugleich auch Regelungs- bzw. Inhaltsadressat des belastenden Verwaltungsakts ist (vgl. OVG NW, Beschluss vom 12. Oktober 2015 - 12 A 2011/15 -, juris Rn. 2; BayVGH, Urteil vom 18. Juli 2012 - 22 A 09.40036 -,juris Rn. 19; Happ, in: Eyermann, VwGO, Kom- 
mentar, 15. Aufl. 2019, § 42 Rn. 91; Schmidt-Kötters, in: Posser/ Wolff, BeckOK VwGO, Stand: 1. Juli 2017, § 42 Rn. 173). Anhaltspunkte, die nach einer denkbaren Betrachtungsweise dafür streiten könnten, dass die Mutter des Klägers - neben ihrem Ehemann F. - auch Inhaltsadressatin des Bescheides gewesen ist, lassen sich nichtfeststellen. Hierbei ist zu berücksichtigen, dass es sich bei der Eintragungsverfügung um einen Verwaltungsakt handelt, der konstitutivlediglich für und gegen jeden potentiellen Ausführungsberechtigten wirkt (vgl. VG Hannover, Urteil vom 9. Juni 1989 - 6 A 69/87 -, NVwZ-RR 1991, 643;ebenso VG Berlin, Urteil vom 9. Februar 1994 - 1 A 29.92 -, juris Rn. 12; im Anschluss daran Bernsdorff/Klein-Tebbe, Kulturgutschutz in Deutschland,Kommentar, 1996, § 1 KultgSchG Rn. 71), selbst aber in keiner denkbaren Weise eine Verfügungsbefugnis seines Adressaten zu begründen vermag.Weder den Akten noch dem bisherigen Vorbringen des Klägers lässt sich auch nur ansatzweise entnehmen, dass seine Mutter zum Kreis der potentiell Ausführungsberechtigten gezählt hat; er erachtet dies lediglich - und in hiernicht ansatzweise genügender Weise - nicht als ausgeschlossen. An diesem Befund ändert auch die (etwaige) Vorstellung der den Bescheid erlassenden Behörde über die „dingliche“ Stellung der Rechtsvorgängerin des Klägersgegenüber dem (hier noch streitgegenständlichen) Kulturgut nichts. Frau I... istinfolgedessen auch nicht etwa - wie der Kläger unter Hinweis auf die Betrachtungsweise der Behörde zu bedenken gibt - zur "richtigen“ Adressatinim Sinne des Kulturgutschutzgesetzes vom 8. Juli 1999 geworden.

[13] Eine Rechtsverletzung der Rechtsvorgängerin des Klägers, auf die sich der Kläger berufen könnte, erschließt sich auch nicht aus dem Umstand, dass ihr - wie mit dem Rechtsbehelf vorgetragen - das Eigentum oder ein im Sinne des Kulturgutschutzgesetzes vom 8. Juli 1999 gleichermaßen bedeutsames Verfügungsrecht an den eingetragenen Kunstwerken gewissermaßen unterstellt worden sei und sie mit den daraus erwachsenden Konsequenzen konfrontiert werde. Dies ändert nichts daran, dass Frau I..., die erkennbar über keine potentielle Ausfuhrberechtigung bezogen auf das streitgegenständliche Werk verfügt hat, nicht nachteilig von der Regelungswirkung des Bescheides betroffen sein konnte; diese Wirkung ging bezogen auf die Mutter des Klägers offenkundig ins Leere. Dass der bloße Rechtsschein insoweit nicht zu genügen vermochte, ist vom Verwaltungsgericht in der angefochtenen Entscheidung näher begründet worden. Auf diese Argumentation geht der Rechtsbehelf ebenso wenig näher ein wie auf die erstinstanzliche Annahme, dass ein etwaigerbelastender Rechtsschein zu Lasten der Mutter des Klägers ihm gegenüber nichtmehr fortwirke.

[14] Der Kläger kann die von ihm angenommene Klagebefugnis fernernicht damit überzeugend begründen, dass es für inn möglich erscheine, das Eigentum oder eine aus dem Eigentumsrecht fließende Verfügungsbefugnis andem streitgegenständlichen Kunstwerk zu erlangen. Ungeachtet der Frage, ob eine entsprechende zukünftige Stellung überhaupt geeignet ist, die Möglichkeit einer Rechtsverletzung im vorliegenden Fall nahezulegen, vermittelt das Zulassungsvorbringen keiner- lei Anhaltspunkte dafür, dass der Kläger als Rechtsnachfolger seiner Mutter (nicht als derjenige seines Vaters) die (erhoffte) Rechtsstellung zukünftig innehaben wird. Die Hoffnungen des Klägers erschöpfen sich insoweit in nicht näher unterlegten Spekulationen. Fehlt es aber an erkennbaren Umständen, die die Annahme stützen könnten, dass die Rechtsvorgängerin des Klägers Eigentümerin oder ansonsten zur Ausfuhr des besagten Werks Berechtigte gewesen ist, erschließt sich auch nicht, wie der Kläger in die für die Begründung einer Klagebefugnis erforderliche Rechtsstellung auf nachvollziehbare Weise gelangen sollte.

[15] Als substanzlos erweist sich auch der Hinweis des Klägers, Rechtsnachfolger müssten schon deshalb klagen können, weil der Bescheid sie auch in ihrer Möglichkeit behinderte, das Eigentum zu erlangen. Denn eine derartige Beschränkung regelte das Kulturgutschutzgesetz vom 8. Juli 1999 nicht. Abgesehen davon erschließt sich auch insoweit nicht plausibel, aus welchen Gründen es möglich erscheinen sollte, dass der Kläger jedenfalls als Rechtsnachfolger seiner Mutter das Eigentum oder eine daraus fließende Verfügungsbefugnis bezüglich des streitgegenständlichen Werks erlangen könnte.

[16] Soweit der Kläger meint, dem Beklagten als Urheber des angefochtenen Bescheids obliege die Darlegungs- und Beweislast für die Voraussetzungen der Eintragung, mithin also auch für die Verfügungsberechtigung der Rechtsvorgängerin des Klägers, führt diese Erwägung nicht weiter. Der Kläger übersieht, dass sich die Frage der Verfügungsberechtigung bereits auf der Ebene der Zulässigkeit stellt, da es hier um eine gerade mit dieser Verfügungsberechtigung verbundene subjektive Rechtsverletzung geht; die Darlegungs- und Beweislast insoweit trägt derjenige, der sich der Möglichkeit einer subjektiven Rechtsverletzung (als Voraussetzung für die Annahme einer Klagebefugnis) berühmt (vgl. zur Darlegungs- und Beweislast bezüglich der Voraussetzungen des $\S 42$ Abs. 2 VwGO Happ, in: Eyermann, VwGO, Kommentar, 15. Aufl. 2019, § 42 Rn. 113 unter Hinweis auf BVerwG, Urteil vom 27. November 1996 - BVerwG 11 A 100.95 -, juris Rn. 34). Ob die Voraussetzungen der Eintragung vorliegen, ist eine Frage der Begründetheit, die im hier erörterten Zusammenhang freilich ohne Belang ist.

[17] bb) Ohne Erfolg wendet sich der Kläger (hier als Rechtsnachfolger seines Vaters und seiner Mutter, der vormaligen Kläger zu 1. und 2.) gegen die erstinstanzliche Würdigung, der angefochtene Bescheid vom 22. Juni 2011 sei rechtmäßig, soweit dort die Voraussetzungen für die Eintragung des von George Grosz stammenden Werks „Brillantenschieber im Café Kaiserhof" in das "Verzeichnis national wertvollen Kulturgutes" bejaht werden

[18] (1) Keine ernstlichen Zweifel zeigt der Kläger auf, soweit er darauf hinweist, es sei umfassend vorgetragen worden, dass das gesamte Verfahren über die Unterschutzstellung der Werke "manipulativ und aus sachfremden Erwägungen" eingeleitet und geführt worden sei. 
[19] (a) Die Bezugnahme des Klägers auf das Vorbringen in der Klageschrift vom 30. Juni 2011 (dort S. 2 bis 4) sowie in den Schriftsätzen seines Prozessbevollmächtigten vom 16. September 2014 (dort S. 2 bis 6) und vom 25. November 2014 (dort S. 1 bis 6) muss unergiebig bleiben. Abgesehen davon, dass ein solcher Verweis die nach § 124a Abs. 4 S. 4 VwGO notwendige Auseinandersetzung mit den Gründen der angefochtenen Entscheidung nicht ersetzen kann und der Kläger damit seinen Darlegungsobliegenheiten nicht entspricht, legen die dort enthaltenen Ausführungen auch keine ernstlichen Richtigkeitszweifel nahe. Das Verwaltungsgericht hat sich mit den dort - unter dem Aspekt der angeblich missbräuchlichen Führung des Eintragsverfahrens durch den Beklagten -angesprochenen Fragen, insbesondere zu den mit der Eintragung vermeintlich verfolgten verfahrensfremden Zielen, zu den Kenntnissen des Sachverständigenausschusses und zu dem Vorwurf einer Ungleichbehandlung im Verhältnis zu anderen Werken, näher befasst und ist auch auf die klägerseits ferner monierten Verfahrensmängel eingegangen (vgl. S. 10 ff. des Urteils). Auf diese Erwägungen geht das Rechtsbehelfsvorbringen nicht substantiiert ein und setzt auch die mit ihm durch den Verweis hervorgehobenen Ausführungen des Klägers im Rahmen des erstinstanzlichen Verfahrens nicht in Bezug zu diesen erstinstanzlichen Überlegungen. So wird die Würdigung des Verwaltungsgerichts nicht ansatzweise in Frage gestellt.

[20] Entsprechendes gilt für den ergänzenden Hinweis des Klägers, "dass sachfremde Erwägungen zur Verfahrensdurchführung in den gesamten Verwaltungsvorgängen" dokumentiert seien, "dort insbesondere auch auf den ersten Seiten - Seiten 1-10". Auch dieses Vorbringen steht nicht im Einklang mit den nach § 124a Abs. 4 S. 4 VwGO zu beachtenden Darlegungsanforderungen. Der Senat war nicht gehalten, sich der Aufgabe zu unterziehen, die Verwaltungsvorgänge auf vermeintliche "sachfremde Erwägungen“ durchzusehen und sie den Erwägungen des Verwaltungsgerichts gegenüber zu stellen, um so ernstliche Zweifel an der Richtigkeit des angefochtenen Urteils aufzudecken, wohl aber der Kläger; ihr ist er aber auch insoweit nicht gerecht geworden.

[21] (2) Soweit der Kläger die erstinstanzliche Würdigung der möglicherweise fehlerhaften Besetzung des nach § 2 Abs. 2 KultgSchG im Vorfeld der Eintragung nach $\S 1$ Abs. 1 KultgSchG anzuhörenden Sachverständigenausschusses in Frage zu stellen sucht, gelingt dies nicht.

[22] (a) Das Verwaltungsgericht hat insbesondere dahinstehen lassen, ob die Bedenken gegen die Besetzung des Ausschusses im Mai 2011, die wegen der Teilnahme des Direktors der S... Dr. T... bestehen könnten, von durchgreifender Natur sind, weil ein entsprechender Fehler gem. § 46 VwVfG unbeachtlich wäre; dessen Voraussetzungen lägen hier vor. Zur Begründung hat es ausgeführt: Dass die Eintragungsverfügung nicht nichtig wäre, folge bereits aus $\S 44$ Abs. 3 Nr. 3 VwVfG, wonach ein Verwaltungsakt nicht deshalb nichtig sei, weil ein durch Rechtsvorschrift zur Mitwirkung berufener Ausschuss den für den Erlass des Verwaltungsaktes vorgeschriebenen Beschluss nicht gefasst habe oder nicht beschlussfähig gewesen sei. Eine Beeinflussung der Eintragungsentscheidung in der Sache erscheine bereits deshalb ausgeschlossen, weil die streitgegenständlichen Beschlüsse ausweislich des Ausschuss-Protokolls einstimmig bzw. mit lediglich einer Stimmenthaltung gefasst worden seien. Zudem sei lediglich die Anhörung des Sachverständigenausschusses gesetzlich vorgeschrieben, eine Bindung der Behörde an das Votum der Sachverständigen bestehe nicht. Mithin fehlte es an einer rechtlich relevanten Kausalität etwaiger Besetzungsfehler im Hinblick auf die angefochtene Entscheidung. Die Entscheidung über die Eintragung von Kulturgut sei keine Ermessens-, sondern eine gebundene Entscheidung; auch verbleibe der Behörde keinerlei Beurteilungsspielraum. Mithin sei, soweit die Eintragungsfähigkeit der Kunstwerke festgestellt werde, für eine andere Entscheidung kein Raum mehr (vgl. S. 11 des Urteils; in juris abgedruckt unter Rn. 33).

[23] (b) Mit seinem Zulassungsvorbringen greift der Kläger lediglich die Erwägung des Verwaltungsgerichts an, das eine Beeinflussung der Eintragungsentscheidung in der Sache bereits deshalb ausgeschlossen hat, weil die streitgegenständlichen Beschlüsse ausweislich des Ausschuss-Protokolls einstimmig bzw. mit lediglich einer Stimmenthaltung gefasst worden seien. Auf die - erstinstanzlich als weitere selbständig entscheidungstragende Erwägung kenntlich gemachte („Zudem ... “) - fehlende Bindung der für die Eintragung nach $\S 1$ Abs. 1 KultgSchG zuständigen Behörde an den Beschluss des Sachverständigenausschusses geht er hingegen nicht ein. Entsprechendes gilt für die Ausführungen des Verwaltungsgerichts zur Einordnung der Eintragung als gebundene Entscheidung ohne jeglichen Beurteilungsspielraum. Vor diesem Hintergrund erweisen sich die daran anknüpfenden Mutmaßungen zu einer Manipulation des Entscheidungsvorgangs durch die Teilnahme des Direktors der S... als unergiebig.

[24] (3) Die klägerseits vorgetragene Kritik gegen die erstinstanzliche Annahme, das Verfahren sei nicht deshalb rechtsmissbräuchlich, weil es von der Einleitung im Jahr 2006 bis zur Eintragung der Kunstwerke im Jahr 2011 über fünf Jahre gedauert habe, führt den Berufungszulassungsantrag ebenfalls nicht zum Erfolg. Soweit der Kläger meint, das Verwaltungsgericht nehme letztlich an, dass das Verfahren „noch nicht übermäßig lange gedauert habe", verfehlt er den Kern der gerichtlichen Argumentation, in der maßgeblich darauf abgehoben wird, dass die gerügte Dauer allein keine Rechtfertigung für die Annahme biete, dass das Verfahren rechtsmissbräuchlich in die Länge gezogen worden sei. Die Dauer an sich bewertet das Verwaltungsgericht nicht. Von einem Widerspruch der angefochtenen Entscheidung zu dem Beschluss der 1. Kammer des Verwaltungsgerichts vom 27. Juli 2011 (- VG 1 K 77.11 -) kann mithin - entgegen der Ansicht des Klägers - keine Rede sein. Der klägerseits angeregten Beiziehung der Akten des zuvor erwähnten Verfahrens durch den Senat bedurfte es vor diesem Hintergrund nicht. 
[25] cc) Soweit der Kläger mit seinen Schreiben vom 22. und 29. Juli 2015 Gesichtspunkte vorgetragen hat, die über die Erläuterung und Vertiefung rechtzeitig vorgebrachter Gründe hinausgehen, ist sein Vorbringen unbeachtlich. Da die Zulassungsgründe gem. § 124 a Abs. 4 S. 4 VwGO innerhalb von zwei Monaten nach Zustellung des Urteils darzulegen sind, können bei der Entscheidung über die Zulassung der Berufung grundsätzlich nur solche Umstände beachtet werden, auf die sich der Rechtsbehelfsführer fristgerecht berufen hat.

[26] b) Der weiterhin geltend gemachte Zulassungsgrund der Divergenz ( $\$ 124$ Abs. 2 Nr. 4 VwGO) ist nicht in der gebotenen Weise dargelegt.

[27] aa) Die Divergenz ist nur dann hinreichend bezeichnet, wenn der Zulassungsantrag einen inhaltlich bestimmten, die angefochtene Entscheidung tragenden abstrakten Rechtssatz benennt, mit dem die Vorinstanz einem in der Rechtsprechung des Oberverwaltungsgerichts, des Bundesverwaltungsgerichts, des gemeinsamen Senats der obersten Gerichtshöfe des Bundes oder des Bundesverfassungsgerichts tragenden Rechtssatz in Anwendung derselben Rechtsvorschrift widersprochen hat (vgl. BVerwG, Beschluss vom 18. September 2006 - BVerwG 10 B 55.06 -, juris Rn. 7 zu § 132 Abs. 2 Nr. 2 VwGO m.w.N.; OVG BlnBbg, Beschluss vom 16. Oktober 2017 - OVG 10 N 57.17 -, juris Rn. 5).

[28] bb) Gemessen an diesen Anforderungen ist die Divergenzrüge schon deshalb nicht prozessual ordnungsgemäß dargelegt, weil der Rechtsbehelf keine in der angefochtenen Entscheidung enthaltenen Rechtssätze aufzeigt, die einem Rechtssatz in dem klägerseits allein in Bezug genommenen Urteil des Bundesverwaltungsgerichts vom 24. November 2011 (- BVerwG 7 C 12.10 -, juris) widersprechen.

[29] Es lässt sich auf der Grundlage des Zulassungsvorbringens auch nicht erkennen, dass der in dem zitierten höchstrichterlichen Urteil und an die Gesetzesbegründung (vgl. BT-Drs. 2/76, S. 7) anknüpfende - Rechtssatz, die Entscheidung über die Eintragung erfordere eine sorgfältige Prüfung, ob das betreffende Kulturgut tatsächlich als national wertvoll einzustufen sei, weil seine Abwanderung aus dem Gebiet der Bundesrepublik Deutschland einen wesentlichen Verlust für den gesamten deutschen Kulturbesitz bedeuten würde (BVerwG, Urteil vom 24. November 2011 - BVerwG 7 C 12.10 -, juris Rn. 52), mit den Prämissen des Verwaltungsgerichts nicht im Einklang steht. Das Gegenteil ist der Fall. Die Entscheidungen stimmen in ihrem rechtlichen Ansatz überein. In dem erstinstanzlichen Urteil wird zum Maßstab des § 1 Abs. 1 KultgSchG - übrigens orientiert an der Rechtsprechung desselben Senats des Bundesverwaltungsgerichts (vgl. Urteil vom 27. Mai 1993 - BVerwG 7 C 33.92 -, juris Rn. 10) - ausgeführt: Einen wesentlichen Verlust bereite dem deutschen Kulturbesitz nur die Abwanderung bedeutsamer und national wertvoller Werke. Die den Begriff des national wertvollen Kulturgutes prägenden Merkmale ließen sich abstrakt nicht abschließend bestimmen; sie seien vielmehr mit Blick auf die im Einzelfall für eine Eintragung anstehenden Objekte im Rahmen einer Gesamtschau zu ermitteln. Dabei fielen die künstlerische Eigenart, der (kunst-)historische Rang und der kulturelle Wert der Objekte ebenso ins Gewicht wie ihre Einzigartigkeit oder Seltenheit sowie ihre Bedeutung für die kulturelle Entwicklung in Deutschland (S. 14 des Urteils; in juris abgedruckt unter Rn. 40). Dass das Verwaltungsgericht im Gegensatz zu dem Urteil des Bundesverwaltungsgerichts vom 24. November 2011 (aaO) von einer "erleichterten Unterschutzstellung" von Kulturgütern ausgeht, wie der Kläger meint, lässt sich aus diesen Erwägungen nicht ablesen. Wie das Bundesverwaltungsgericht in der Klägerweise zitierten Entscheidung nimmt auch das Verwaltungsgericht bei seiner Prüfung eine Gesamtschau vor und betrachtet dabei maßstäblich die Wirkung des Verlusts für den gesamten deutschen Kulturbesitz als Voraussetzung, wie insbesondere die Formulierung „Einzigartigkeit oder Seltenheit sowie ihre Bedeutung für die kulturelle Entwicklung in Deutschland" (Hervorhebung durch den Senat) belegt. Die von dem Kläger im Weiteren für die von ihm vertretene Divergenzannahme herangezogenen Erwägungen des Verwaltungsgerichts (vgl. dazu den Schriftsatz des Prozessbevollmächtigten des Klägers vom 26. April 2015, S. 10 f.) sind bereits Teil der Subsumtion unter den Maßstab (s. S. 14 und 19 des Urteils). Eine damit verbundene (vermeintlich) fehlerhafte Rechtsanwendung vermag eine Abweichung im Sinne des $\S 124$ Abs. 2 Nr. 4 VwGO indes nicht zu begründen

[30] Die von dem Kläger im Weiteren für die von ihm vertretene Divergenzannahme herangezogenen Erwägungen des Verwaltungsgerichts (vgl. dazu den Schriftsatz des Prozessbevollmächtigten des Klägers vom 26. April 2015, S. 10 f.) sind bereits Teil der Subsumtion unter den Maßstab (s. S. 14 und 19 des Urteils). Eine damit verbundene (vermeintlich) fehlerhafte Rechtsanwendung vermag eine Abweichung i. S. d. § 124 Abs. 2 Nr. 4 VwGO indes nicht zu begründen.

[31] Nach alledem kann dahinstehen, ob selbst eine bestehende Divergenz es überhaupt rechtfertigte, die Berufung zuzulassen. Insofern bestehen Bedenken, weil die im vorliegenden Zusammenhang angesprochenen Auslegungsprobleme ausgelaufenes oder auslaufendes Recht betreffen [s. näher zum Vorliegen ausgelaufenen Rechts den nachfolgenden Abschnitt c); eine Divergenzzulassung in solchen Fällen ablehnend BVerwG, Beschlüsse vom 15. Oktober 2009 - BVerwG 1 B 3.09 -, juris Rn. 9, und vom 23. Dezember 1998 - BVerwG 2 B 106.98 -, juris Rn. 2; a.A. Beschluss vom 27. Februar 1997 - BVerwG 5 B 145.96 -, juris Rn. 1)].

[32] C) Die Sache hat ferner keine grundsätzliche Bedeutung ( $\S$ 124 Abs. 2 Nr. 3 VwGO).

[33] aa) Eine Rechtssache hat grundsätzliche Bedeutung, wenn sie eine abstrakte, in dem zu entscheidenden Fall erhebliche Rechts- oder Tatsachenfrage mit einer über den Einzelfall hinausgehenden allgemeinen Bedeutung aufwirft, die im Interesse der Einheitlichkeit der Rechtsprechung oder im Interesse 
der Rechtsfortbildung in einem Berufungsverfahren geklärt werden muss. Das Darlegungserfordernis des $\S 124 a$ Abs. 4 S. 4 VwGO verlangt daher zur Begründung einer grundsätzlichen Bedeutung neben der Bezeichnung der Frage Ausführungen zur Klärungsbedürftigkeit, Klärungsfähigkeit und zur Entscheidungserheblichkeit der aufgeworfenen Rechts- oder Tatsachenfrage (s. dazu Senatsbeschluss vom 29. Januar 2019 - OVG 10 N 67.16 -, juris Rn. 18).

[34] bb) Die von dem Kläger zu der in der Rechtsprechung des Bundesverwaltungsgerichts (Urteil vom 24. November 2011 BVerwG 7 C 12.10 -, juris Rn. 52) vertretenen Auslegung des § 1 Abs. 1 S. 1 KultgSchG und ihrer weiteren Konkretisierung im Hinblick auf das Tatbestandsmerkmal „wesentlicher Verlust für den deutschen Kulturbesitz" aufgeworfenen Fragen,

[35]- „Kann auf die Kriterien der Kultusministerkonferenz, alter Fassung, auf die hier zurückgegriffen wird, in der vorliegenden Form zurückgegriffen werden?",

[36] - „Von welcher Bedeutung ist die Weltreputation?" und

[37] - „Welcher Maßstab ist an den möglichen Seltenheitswert anzulegen, aus welchen Bezügen ist er herzuleiten, beispielsweise aus der Seltenheit im gesamten deutschen Kulturbesitz, der Seltenheit bezogen auf den Künstler oder der Seltenheit in der Welt?"

[38] verleihen der Rechtssache keine grundsätzliche Bedeutung. Es erscheint mit Blick auf die erforderliche Gesamtschau bei der Bestimmung der Eintragungsvoraussetzungen (vgl. BVerwG, Urteil vom 27. Mai 1993 - BVerwG 7 C 33.92 -, juris Rn. 10) schon zweifelhaft, ob sich bezogen auf die besagten Fragen überhaupt allgemeine Aussagen treffen lassen. Dies kann dahinstehen, weil die aufgeworfenen Fragen die Interpretation von ausgelaufenem oder auslaufendem Recht betreffen. An die Stelle des Kulturschutzgutgesetzes vom 8. Juli 1999 ist das Gesetz zum Schutz von Kulturgut (Kulturgutschutzgesetz - KGSG) vom 31. Juli 2016 (BGBI. I S. 1914) getreten, das insbesondere die Voraussetzungen der Eintragung in das Verzeichnis national wertvollen Kulturgutes in $\S 7$ KGSG abweichend von $\S 1$ Abs. 1 S. 1 KultgSchG regelt.

[39] Eine Rechtsfrage, die sich auf ausgelaufenes, auslaufendes oder nur übergangsweise geltendes Recht bezieht, hat regelmäßig keine grundsätzliche Bedeutung, da § 124 Abs. 2 Nr. 3 VwGO eine richtungsweisende Klärung für die Zukunft herbeiführen soll. Eine Revisionszulassung kommt deshalb nur ausnahmsweise in Betracht, wenn sich die Frage für die Nachfolgeregelung offensichtlich in gleicher Weise stellt oder wenn ihre Beantwortung für einen nicht überschaubaren Personenkreis in nicht absehbarer Zukunft von Bedeutung ist und dies substantiiert dargelegt wird (stRspr, BVerwG, Beschluss vom 28. August 2018 - BVerwG 3 B 28.17 -, juris Rn. 10 m.w.N.). Beides ist hier nicht der Fall.
[40] Die Nachfolgeregelung in $\S 7$ KGSG weist gegenüber $\S 1$ Abs. 1 S. 1 KultgSchG erhebliche Veränderungen auf, auch wenn der Gesetzgeber mit der neuen Regelung an $\S 1$ Abs. 1 KultgSchG anzuknüpfen sucht (vgl. Begründung zum Gesetzentwurf der Bundesregierung, BT-Drs. 18/7456, S. 67 f.). Während nach $\S 1$ Abs. $1 \mathrm{~S}$. 1 KultgSchG Kunstwerke und anderes Kulturgut einzutragen waren, wenn "deren Abwanderung aus dem Geltungsbereich dieses Gesetzes einen wesentlichen Verlust für den deutschen Kulturbesitz bedeuten würde“, ist Kulturgut nach $\S 7$ Abs. 1 S. 1 KGSG „in ein Verzeichnis national wertvollen Kulturguts einzutragen, wenn 1. es besonders bedeutsam für das kulturelle Erbe Deutschlands, der Länder oder einer seiner historischen Regionen und damit identitätsstiftend für die Kultur Deutschlands ist und 2. seine Abwanderung einen wesentlichen Verlust für den deutschen Kulturbesitz bedeuten würde und deshalb sein Verbleib im Bundesgebiet im herausragenden kulturellen öffentlichen Interesse liegt ". Danach ist nicht zu ersehen, dass sich die vom Kläger als grundsätzlich bedeutsam erachteten Fragen offensichtlich in gleicher Weise bei der Auslegung der neustrukturierten und mit einer Reihe von weiteren Tatbestandsmerkmalen versehenen Regelung des $\S 7$ Abs. 1 KGSG stellen.

[41] Dass die Beantwortung der klägerseits aufgeworfenen Fragen noch für einen nicht überschaubaren Personenkreis in nicht absehbarer Zukunft von Bedeutung sein könnte, lässt sich ebenfalls nicht erkennen.

[42] d) Die Berufung ist schließlich auch nicht wegen des klägerseits geltend gemachten Zulassungsgrundes des $\S 124$ Abs. 2 Nr. 5 VwGO zuzulassen. Verfahrensmängel, auf denen die Entscheidung beruhen könnte, liegen nicht vor bzw. sind nicht im genügenden Maße dargelegt.

[43] aa) Die geltend gemachten Gehörsrügen greifen nicht.

[44] (1) Das Gebot rechtlichen Gehörs gem. Art. 103 Abs. 1 GG verpflichtet das Gericht dazu, die Ausführungen der Verfahrensbeteiligten zur Kenntnis zu nehmen und bei seiner Entscheidung zu berücksichtigen. Davon, dass das Gericht ihm unterbreitetes Vorbringen auch tatsächlich zur Kenntnis nimmt und berücksichtigt, ist grundsätzlich auszugehen (vgl. BVerfG, Beschluss vom 24. Februar 2009 - 1 BvR 188/09 -, NVwZ 2009, 580 m.w.N.). Erforderlich ist es auch nicht, dass das Gericht sich mit jedem Vorbringen der Beteiligten in den Gründen seiner Entscheidung ausdrücklich auseinandersetzt (BVerwG, Beschlüsse vom 1. April 2015 - BVerwG 4 B 10.15 -, juris Rn. 2 und vom 24. November 2011 - BVerwG 8C 13.11 -, juris Rn. 2). Vielmehr ist dem Anspruch auf rechtliches Gehör Genüge getan, wenn das Gericht sich in seiner Begründung mit dem für die Entscheidung erheblichen Kern des Beteiligtenvorbringens jedenfalls zu den Fragen, die für das Verfahren von zentraler Bedeutung sind, auseinandersetzt. Erst wenn sich im Einzelfall klar ergibt, dass das Gericht dieser Pflicht nicht nachgekommen ist, ist der Anspruch auf rechtliches Gehör verletzt (vgl. OVG Berlin-Brandenburg, Beschluss vom 4. Dezember 2012 - OVG 11 RS 3.12, OVG 11 RM 1.12 
-, juris Rn. 2). Es ist daher verfehlt, aus der Nichterwähnung einzelner Begründungsteile eines Beschwerdevorbringens in den gerichtlichen Entscheidungsgründen zu schließen, das Gericht habe sich nicht mit den darin enthaltenen Argumenten befasst (BVerfG, Beschluss vom 15. April 1980 - 1 BvR 1365/78 - BVerfGE 54, 43, 46 m.w.N.; s. zu alledem auch Senatsbeschluss vom 26. November 2014 - OVG 10 N 27.12 -, juris Rn. 9).

[45] (2) Hieran gemessen ist ein Gehörsverstoß nicht feststellbar.

[46] (a) Die mit dem Rechtsbehelf vorgetragene Rüge, das Verwaltungsgericht habe bei der Würdigung der Klagebefugnis seinen Vortrag nicht zur Kenntnis genommen, dass der Beklagte im Eintragungsverfahren immer davon ausgegangen sei, dass die Rechtsvorgängerin des Klägers Eigentümerin eingetragenen Werkes gewesen sei, und als Urheber des Bescheides auch die Beweislast für die Eintragungsvoraussetzungen trage, führt nicht weiter, weil diese Argumente - wie bereits erörtert - nicht zu überzeugen vermögen und die angefochtene Entscheidung auf einem etwaigen Gehörsverstoß insoweit nicht beruhen könnte.

[47] (b) Soweit der Kläger auf sein Vorbringen in der Klageschrift vom 30. Juni 2011 (dort S. 2 bis 4) sowie in den Schriftsätzen seines Prozessbevollmächtigten vom 16. September 2014 (dort S. 2 bis 6) und vom 25. November 2014 (dort S. 1 bis 6) verweist, hat der Senat bereits im Rahmen seiner Ausführungen zu dem klägerseits geltend gemachten Berufungszulassungsgrund ernstlicher Zweifel an der Richtigkeit der angefochtenen Entscheidung darauf hingewiesen, dass sich das Verwaltungsgericht mit den in den Schriftsätzen angesprochenen Problemstellungen auseinandergesetzt hat, insbesondere mit der Frage der Verfolgung eintragungsfremder Ziele durch den Beklagten, ferner mit den Kenntnissen des Sachverständigenausschusses von den Umständen der Verkaufsverhandlungen über die von der Eintragung erfassten Werke zwischen F... und der Berlinischen Galerie, mit dem Vorwurf einer Ungleichbehandlung im Verhältnis zu anderen Werken sowie mit der Dauer und möglichen Fehlern des Eintragungsverfahrens.

[48] (c) Dass das Verwaltungsgericht keine näheren Erwägungen zu den klägerseits im erstinstanzlichen Verfahren angesprochenen Fragen, ob die im Vorfeld der Sitzungen des Sachverständigenausschusses erstellten Gutachten von der nötigen Neutralität getragen gewesen seien und ob der Eintragung des Werkes die sachfremde Erwägung zugrunde gelegen habe, mit ihr zu erreichen, den Vater des Klägers von den bei einer Veräußerung der Werke anfallenden Steuern zu befreien, ist nicht zu beanstanden. Diesen Fragen musste das Verwaltungsgericht schon eingedenk des von ihm eingenommenen Rechtsstandpunktes nicht nachgehen, dass angesichts der offenbaren Bedeutung der in Rede stehenden Künstler tragfähige und nachvollziehbare Anhaltspunkte dafür vorgelegen hätten, dass die betreffenden Werke national wertvoll i.S.d. § 1 Abs. 1 S. 1 KultgSchG sein könnten. Dem Berufungszulassungsvorbringen lässt sich nicht entnehmen, dass der Kläger diese Anhaltspunkte bezogen auf George Grosz und dessen Werk „Brillantenschieber im Café Kaiserhof" etwa negierte.

[49] bb) Auch die Aufklärungsrügen, mit denen der Kläger in der Sache eine Verletzung von $\S 86$ Abs. 1 VwGO geltend macht, verfangen nicht.

[50] (1) Eine solche Rüge kann nur Erfolg haben, wenn sie schlüssig aufzeigt, dass das Gericht aufgrund seiner Rechtsauffassung Anlass zur weiteren Aufklärung hätte sehen müssen. Mit dem Zulassungsantrag muss weiter substantiiert dargetan werden, hinsichtlich welcher tatsächlichen Umstände Aufklärungsbedarf bestanden hat, welche für geeignet und erforderlich gehaltenen Aufklärungsmaßnahmen hierfür in Betracht gekommen wären und welche tatsächlichen Feststellungen bei der Durchführung der vermissten Sachverhaltsaufklärung voraussichtlich getroffen worden wären. Außerdem muss dargelegt werden, dass bereits im Verfahren vor dem Verwaltungsgericht in der mündlichen Verhandlung auf die Vornahme der Sachverhaltsaufklärung, deren Unterbleiben nunmehr beanstandet wird, hingewirkt worden ist, oder dass sich dem Gericht die bezeichnete Ermittlung auch ohne ein solches Hinwirken von sich aus hätte aufdrängen müssen (so zu alledem Senatsbeschluss vom 29. August 2018 - OVG 10 N 13.15 -, juris Rn. 21 m.w.N.).

[51] (2) Diesen Anforderungen genügt das Vorbringen des Klägers nicht. Sofern er rügt, dass das Verwaltungsgericht seinen "umfangreichen Beweisantritten“ verfahrensfehlerhaft nicht nachgegangen sei, dringt er damit nicht durch. Da sein Prozessbevollmächtigter es unterlassen hat, entsprechende Beweisanträge in der mündlichen Verhandlung vor dem Verwaltungsgericht zu formulieren, kann es im vorliegenden Zusammenhang nur darauf ankommen, ob es sich dem Verwaltungsgericht aufdrängen musste, den Beweisanregungen nachzugehen. Das ist indes nicht der Fall. Eine Beweiserhebung durch Vernehmung der Mitglieder des zuständigen Sachverständigenausschusses, wie klägerseits in den Schriftsätzen vom 16. September 2014 (dort auf S. 5) und 25. November 2014 (dort auf S. 3 unten und S. 4 oben) mit Blick auf die dort angesprochenen Themen angeregt, musste sich dem Verwaltungsgericht nach seiner Rechtsauffassung nicht aufdrängen, weil es mit Blick auf den Inhalt des Ausschussprotokolls vom 18. Mai 2011 davon ausgegangen ist, dass die Ausschussmitglieder Kenntnis von den zivilrechtlichen Streitigkeiten und den Verkaufsverhandlungen zwischen F... und dem Beklagten hatten, und es ihm fernliegend erschien, dass der Sachverständigenausschuss bewusst über die Erwerbsaussichten des Beklagten getäuscht worden ist. Abgesehen davon lässt sich dem Berufungszulassungsvorbringen nicht entnehmen, dass die Beweisanregungen auf mehr als bloßen Vermutungen und Spekulationen über Manipulationen beruhen, denen die Mitglieder des Sachverständigenausschusses angeblich unterlegen gewesen sein sollen (vgl. die Mutmaßungen auf S. 8 f. des Schriftsatzes vom 26. April 2015); sie erweisen sich vor diesem Hintergrund letztlich nur als Beweiser- 
mittlungsanträge ohne hinreichende substantielle Grundlage, die -wären sie so überhaupt gestellt worden - als unzulässig abzulehnen gewesen wären.

[52] Die Kostenentscheidung folgt mit Blick auf den von den Beteiligten übereinstimmend für erledigt erklärten Teil des Berufungszulassungsverfahrens aus § 161 Abs. 2 S. 1 VwGO und war nach billigem Ermessen zu Lasten des Klägers zu treffen, weil sein Berufungszulassungsantrag wegen der nach dem bisherigen Sach- und Streitstand anzunehmenden Unzulässigkeit seiner Klage auch insoweit [vgl. dazu Abschnitt 2. a)] erfolglos geblieben wäre; im Übrigen ergibt sich die Kostenentscheidung aus $\S 154$ Abs. 2 VwGO.

[53] Die Streitwertfestsetzung beruht auf § 47 Abs. 1 und 3, § 52 Abs. 1 GKG. Im Fall der Eintragung nach dem Kulturgutschutzgesetz ist nach der Senatsrechtsprechung die Hälfte des Verkehrswertes der streitgegenständlichen Kunstwerke anzusetzen (vgl. Senatsbeschluss vom 14. Februar 2012 - OVG 10 L 84.11 -, S. 3 EA). Hinsichtlich der Höhe des Verkehrswertes schließt sich der Senat den Überlegungen des Verwaltungsgerichts in dem für das erstinstanzliche Verfahren ergangenen Streitwertbeschluss an (s. auch VG Berlin, Beschluss vom 22. Januar 2015 - VG 1 K 228.11 -, juris Rn. 64).

[54] Dieser Beschluss ist unanfechtbar (§ 152 Abs. 1 VwGO, § 68 Abs. 1 S. 5 i.V.m. § 66 Abs. 3 S. 3 GKG). 